\title{
A Nonsense Consonant-Vowel-Consonant Word Test to Assess Auditory Processing
}

DOI: $10.3766 /$ jaaa. 16052

\author{
Mariah Nicole Cheyney* \\ Deborah W. Moncrieff $\dagger$
}

\begin{abstract}
Background: Dichotic listening (DL), or how the two ears work together as a team, is often used in the assessment of auditory processing disorders in both children and adults. Currently, the battery of dichotic tests includes stimuli containing words, digits, and nonsense consonant-vowel syllables. Single-syllable nonsense words are of particular use in assessing processing abilities because they can evaluate auditory processing without a listener's dependence on linguistic knowledge. Therefore, nonsense words may assess auditory processes independently of previous vocabulary knowledge.
\end{abstract}

Purpose: This study is designed to assess the clinical applicability and face validity of a nonsense word DL test in a young adult population.

Research Design: This study included an experimental design to investigate the performance of young adult listeners on a Dichotic Nonsense Word (DNW) test spoken by a male and female speaker. The results were compared with one study that investigated young adult listener's performance on dichotic tests of English words.

Study Sample: A total of 100 young adult participants were recruited from the School of Health and Rehabilitation Sciences at the University of Pittsburgh to participate in the study. The participants ranged in age from 20 to 30 , with an average age of 23 , and all participants had normal hearing.

Data Collection and Analysis: DL performance was measured in all participants using the Dichotic Words Test (DWT) and the newly developed DNW test. Kolmogorov-Smirnoff tests of normality were used to assess distribution of right- and left-ear scores. Criterion cutoff scores were determined for the percent correct scores in the nondominant ear and dominant ear and for ear advantage.

Results: Scores were significantly different between the two tests in the right ear, $Z=-8.258, p<0.001$, and in the left ear, $Z=-8.471, p<0.001$. Scores within each test were higher for the right ear than for the left ear, and scores for both ears were significantly lower on the DNW test than for the DWT. Ear advantage scores from the DNW test were significantly larger than those obtained from the DWT. The low and high $95 \%$ criterion cutoff ranges for the DNW test were considerably wider than the $95 \%$ criterion cutoff ranges for the DWT.

Conclusions: Results indicate that the new DNW test may be a useful clinical tool within a test battery for evaluating auditory processing skills independent of vocabulary knowledge.

Key Words: adult, auditory perception, diagnosis, dichotic listening tests/methods, dichotic listening tests/utilization, differential, humans, MeSH terms

Abbreviations: $\mathrm{AMB}=$ amblyaudia; $\mathrm{CV}=$ consonant vowel; $\mathrm{DD}=$ dichotic dysaudia; $\mathrm{DL}=$ dichotic listening; DNW = dichotic nonsense words; DOM = dominant; DWT = dichotic words test; LEA = left-ear advantage; NDOM = nondominant; NEA = no ear advantage; NF = free recall (nonforced); $\mathrm{REA}=$ right-ear advantage; VOT = voice-onset time

${ }^{*}$ Northern Illinois University, Sycamore, IL; †University of Pittsburgh, Pittsburgh, PA

Corresponding author: Mariah Nicole Cheyney, Allied Health and Communication Disorders, Northern Illinois University, Sycamore, IL 60115; Email: mcheyney@niu.edu 


\section{INTRODUCTION}

$\mathrm{D}$ ichotic listening (DL) involves the ability to discriminate differing and simultaneous signals to the ears. Weaknesses in DL are common among individuals with auditory processing disorders (Bellis, 2002; Musiek et al, 2002; DeBonis and Moncrieff, 2008). The two ears work optimally when signals from both ears are alike, and when there are minimal interaural time differences or sound shadow effects, because of the physics of sound and the relative location of the ears (Yost, 2013). When the signals reaching the two ears are different, the competing signals tax the auditory system and force the listener to integrate signals that differ in time and intensity. Standard DL tasks contain stimuli of the same or similar duration that differ in some other aspect, such as phonology. Persons suspected of having an auditory processing disorder should be tested using a variety of stimuli and auditory processes, including tests that use competing signals. DL tests specifically measure the listener's ability to successfully engage in binaural integration, which requires the listener to repeat all information when presentations are made to both ears (Moncrieff, 2006; Moncrieff and Wilson, 2009). DL tasks may force the listener to integrate more stimuli than possible, typically resulting in a difference score between the two ears or an ear advantage.

In a majority of listeners, the right ear is superior to the left ear, and this is referred to as the right-ear advantage (REA). Early research supported a prevalence of the REA especially in right-handed listeners because of a dominance of auditory processing in the left hemisphere where a substantial portion of language is processed in these individuals (Kimura, 1961). The REA has been documented to occur in dichotic tests when the stimuli include digits (Kimura, 1967), words (Roup et al, 2006; Moncrieff, 2011), and consonant-vowels (Hugdahl et al, 2001). The exact prevalence of the REA varies because of factors such as handedness, age, and gender. Recent research suggests a prevalence of a left-ear advantage (LEA), or when the left ear is superior to the right ear, in $18 \%$ of right-handed children and adults when tested with randomized dichotic digits (Moncrieff and Wilson, 2009), in 28\% of children when tested with dichotic words (Moncrieff, 2015), and in $18 \%$ of adults when tested with dichotic words (Hiscock et al, 2000). REA magnitude also decreases with age from childhood to young adulthood (Moncrieff and Wilson, 2009; Moncrieff, 2011), and increases with age from young adulthood to older adulthood (Noffsinger et al, 1996).

Dichotic tasks can be presented to test binaural separation, which assesses the listener's ability to direct attention to one ear or the other. These changes force the listener to process information from one ear at a greater expense to the other, which manipulates the direction of laterality and ultimately the prevalence of REA. In fact, the act of attending can be switched from one ear to the other. For example, directing one ear toward a source of desired attention can in turn affect comprehension performance when in difficult auditory situations (Cherry, 1953). In DL tests, attention is either directed or nondirected. In a nondirected free-recall (NF) condition, the listener is required to repeat back all heard stimuli without attention to order or to ear. Alternatively, dichotic tasks can be manipulated by forcing the listener to repeat the stimulus in the right ear before the left ear or in the left ear before the right, referred to as directed response conditions. Typical listeners show an REA when attention is not directed to either ear, referred to as NF condition, and again when attention is in the forced right condition (Roup et al, 2006). Conversely, an LEA is possible in individuals with an REA when their attention is directed exclusively to the left ear in the forced left condition (Hugdahl and Andersson, 1986).

Open-set single-syllable words place a high verbal workload on the listener, meaning that they are ideal for assessing ear dominance for language (Moncrieff, 2011). Nonsense words and syllables are also useful in assessing verbal processing abilities because they contain commonly used phonemes but they neither place demands on nor benefit from a listener's semantic knowledge. Because of the nonpredictability of nonsense words and syllables, a task including these stimuli is more difficult and will be less likely to display the "ceiling effects" on performance that can occur with highly familiar pairs of digits (Moncrieff and Musiek, 2002; Neijenhuis et al, 2002). The difficulty of these tasks over digits and familiar single-syllable words has been documented using dichotic consonant vowel (CV) stimuli (Hugdahl et al, 2001) and DNW stimuli (Boothroyd and Nittrouer, 1998).

When testing with words, evidence suggests changes in the degree of REA with manipulation of word familiarity (Techentin and Voyer, 2011). Adults tend to rely heavily on phonemic content, or the neighboring syllable and phoneme density, for word recognition. For example, a word will be easier to recognize if a neighboring syllable provides enough information for the listener to have understanding with the help of auditory closure. Word familiarity can affect test performance given that words without lexical neighbors are considerably more difficult to recognize than words with high neighborhood densities. Resultantly, the more difficult listening situations tax the auditory system and force use of the dominant (DOM) ear pathway, increasing the prevalence of REA(Nittrouer and Boothroyd, 1990). Conversely, CV syllables do not contain lexical neighbors and therefore have little dependence on semantic knowledge of any test language. Nonnative English speakers will score comparably on tests using dichotic CV monosyllables in contrast to English words (Keith et al, 1987). Therefore, a nonfamiliar task allows for a test with a "universal design," or a test that can be given across native and nonnative English 
speakers independent of bias because of semantic familiarity. In this study, a new DL task using nonsense words was created using stimuli with little to no phonemic patterns and therefore a universal design.

Currently, no normative data exist for young adult performance on DNW tests. Research examining the feasibility of nonsense stimuli in comparison with standard single-syllable words indicates that performance on tests using nonsense stimuli is significantly worse than performance on tests using English words. For example, these results are documented in the diotic condition in noise in normal-hearing young adults (Boothroyd and Nittrouer, 1988) and in the dichotic condition for normal-hearing young adults (Noffsinger et al, 1994; Findlen and Roup, 2011). The purpose of this study was to observe performance on the newly developed DNW test and to compare results from DNW test with those attained from the Dichotic Words Test (DWT) (Moncrieff, 2011) in the young adult normal hearing listener population. From these comparisons, applicability and clinical usefulness of the DNW test as an evaluation tool for auditory processing in adults will be discussed.

\section{METHODS}

\section{Subjects}

A total of 100 young adult participants were recruited from the School of Health and Rehabilitation Sciences at the University of Pittsburgh to participate in the study. The participants volunteered to participate and were not actively recruited. The participants ranged in age from 20 to 30 , with an average age of 23 . Inclusion criteria included normal hearing (thresholds at 500, 1000, 2000, and 4000 $\mathrm{Hz}$ less than or equal to $20 \mathrm{~dB} \mathrm{HL}$ ) and proficiency in English based on acceptance into a graduate level University program in the United States. All young adults were registered in a graduate school or were faculty members within the School of Health and Rehabilitation Sciences at the time of the study. Exclusion criteria included hearing loss greater than $20 \mathrm{~dB}$ HL for at least one of the tested frequencies listed previously. The participants' history of auditory processing disorder, neurological disorder, or other developmental disorder was unknown. Because of the limited exclusionary criteria, the study results were not intended to create normative data. A total of five participants were excluded from study, three because of hearing loss and two because of equipment malfunction during testing. Of the remaining 95 subjects, 6 were male, and 89 were female. Two participants (one male and one female) were nonnative speakers of English. Those that were nonnative speakers were not excluded given they had English proficiency for acceptance to a graduate university program. The intension was to represent performance in an average population of young adults with normal hearing. Consent was obtained from each individual participant according to the guidelines established by the University of Pittsburgh Institutional Review Board for participants in Pennsylvania.

\section{Materials}

DL performance was measured in all participants using the DWT (Moncrieff, 2011; 2015) and the newly developed DNW test. The DWT contains four lists of word pairs that are presented in a dichotic pattern in which each word pair is matched for length, with temporal alignment of each word onset and offset. The DWT is recorded with a male voice. The DNW test contains four lists of 25 phonetically balanced word pairs. Each word pair was also matched for length in milliseconds. One test version was recorded with a female voice and the other test version with a male voice. Both female and male voices were recorded to determine if fundamental frequency of the stimuli influenced test performance. All nonsense words were aligned at the stimulus onset and offset, equalized in length within $1 / 10$ th of a millisecond. A 3-sec silent interval was inserted between each pair of words to allow time for response. Each word was presented at the same average root-mean-square amplitude.

All words across both tests were recorded and normalized to achieve presentation at the same average root-mean-square amplitude across the test. The presentation level for each participant was a comfortable listening level balanced between the two ears.

\section{Procedures}

Each participant received a hearing evaluation through a two-channel audiometer (Grason Stadler, Model 16). The evaluation was completed under TDH-50 headphones in a sound booth. Hearing thresholds were obtained for $500,1000,2000$, and 4000 in both ears for each participant. All participant thresholds at all frequencies were within normal limits ( $\leq 20 \mathrm{~dB} \mathrm{HL}$ ). After the hearing test, the DWT and the DNW test were administered at a moderately comfortable listening level through a Dell laptop via Bose QuietComfort $\AA^{\circledR} 15$ Acoustic Noise Cancelling ${ }^{\circledR}$ headphones. All participants were asked to listen to the presented word lists at a "loud-but-comfortable" level. If the participant requested volume manipulations, the examiner completed the manipulations.

The DWT was presented using three listening conditions. The participants were asked to repeat both words in (a) an NF condition across one list of 25 word pairs, (b) a directed right condition in which they were asked to repeat both words and say the word heard in the right ear first for another 25 pairs of words, and (c) a directed left (DL) condition in which they were asked to repeat both words and say the word heard in the left ear first for the last 25 pairs of words. All listening conditions for 
the DWT were randomized across participants, and administration of the tests with these procedures was appropriate, given the DWT current clinical applicability. In other words, these tests conditions are most commonly used in the clinical setting.

The DNW test was always presented after the DWT. Half of the participants completed the DNW test female version, and the remaining half completed the DNW test male version, with randomized selection. Administration of the test always began with an NF condition in which the participant repeated only one word. The purpose of this first administration in which the participant was required to only identify one of the two words was to familiarize the participant with the new task and to give the participant an opportunity to practice. The test was then administered in two directed conditions in which they were asked to repeat only the word in the right ear $(\mathrm{R}$ only) and only the word in the left ear (L only), which constitutes a binaural separation task. The final administration of the DNW was done in a standard binaural integration NF condition in which they were instructed to repeat both words (NF). The order of presentation for the R-only-directed and L-only-directed conditions was randomized. Results were scored as correct identification of nonsense words for left and right ears separately in each listening condition. Test procedures for the DNW test differed from the DWT because of the predicted difficulty of the test. The DNW test was predicted to be more difficult because of the lack of semantic content. Despite differences, the ear advantage results were compared within tests for the directed test conditions. Across test comparisons were only made for the NF conditions, which were completed for both tests with the same instructions.

\section{RESULTS}

$\mathrm{T}$ he number of correctly identified words was totaled for each ear after each listening condition was administered and recorded in percent words correct. Ear advantage was calculated as the difference between the ears in percent correct recognition. All scores for ear advantage were first determined in the traditional manner by subtracting the score in the left ear from the score in the right ear. From this information, the prevalence of REA and LEA were determined and were compared across the two types of tests. All results were then converted to DOM ear and nondominant (NDOM) ear without regard to direction to determine ear advantage regardless of which ear was superior. This alternative method of determining ear advantage leads to a larger average value for ear advantage when analyzing dichotic tests using words and digits because the difference score always results in a positive number (Moncrieff, 2011). Ear advantage scores determined by the alternative method were compared between the DNW test and DWT and within each test individually across the three test conditions $(\mathrm{NF}$, directed right, and directed left).

\section{Statistical Analyses}

Kolmogorov-Smirnoff tests of normality (SPSS, IBM Statistics version 22) were used to assess distribution of right and left ear scores. Both ear scores from the DWT were not normally distributed, left-ear statistic $=0.235$ $\mathrm{df}=95, p<0.001$, right-ear statistic $=0.202 \mathrm{df}=95$, $p<0.001$. The right ear score from the DNW test was also not normally distributed, right-ear statistic $=0.123 \mathrm{df}=95$, $p=0.001$, but the left-ear score from the DNW test was normally distributed, left-ear statistic $=0.082 \mathrm{df}=95$, $p=0.123$. Because most of the raw scores were not normally distributed, each score was recalculated with an arcsine transformation. Results from the KolmogorovSmirnoff test of normality on arcsine transformed scores revealed nonnormal distributions for right- and left-ear scores in both tests. Because the arcsine transformed results were also not normally distributed, further statistical analyses were performed on raw scores with nonparametric tests to examine differences across test results. Criterion cutoff scores were determined for the percent correct scores in the NDOM ear and DOM ear and for ear advantage by performing a bootstrap with 1,000 samples at a confidence interval level at the 95 th percentile. Bootstrapping methods have theoretical advantages when applied to non-Gaussian data. In bootstrapping, data are randomly resampled with replacement multiple times (in this case, 1,000 times), and confidence intervals are then drawn from the subsequent data. Confidence intervals from bootstrapping are computed using the z-distribution (for a 95\% confidence interval). The 2.5 and 97.5 percentiles constitute the limits of the 95\% confidence interval (Haukoos and Lewis, 2005; Henderson, 2005).

\section{Ear and Test Differences}

The Kruskal-Wallis test is a nonparametric one-way analysis of variance. The Kruskal-Wallis test was used to measure the effects of ear (right ear, left ear) within the DWT and the DNW test. For this comparison, each test was presented to the participants in the NF condition. Chi-square results demonstrated significant differences in performance between the right and left ears for each test, DWT: $\chi^{2}=8.271, p=0.004$ and DNW: $\chi^{2}=36.168$, $p<0.001$. The Wilcoxon Signed Ranks Test is a nonparametric test to compare pair samples drawn from populations with different mean ranks. It was used to measure the effects of test (DWT and DNW) on individual right and left ear scores in the NF condition. Scores were significantly different between the two tests in the right ear, $Z=-8.258, p<0.001$, and in the left ear, $Z=-8.471$, $p<0.001$. As shown in Figure 1, scores within each test were higher for the right ear than for the left ear and scores 
for both ears were significantly lower on the DNW test than with the DWT.

The Wilcoxon Signed Ranks Test was also used to compare ear advantage scores calculated in the traditional manner (as the difference between right and left ears) between the two tests in the NF condition. The traditionally calculated ear advantage scores were significantly different between the two tests, $Z=-5.523, p<0.001$. Ear advantage scores from the DNW test were larger than those obtained from the DWT as shown by comparing the two gray bars in Figure 2. The prevalence of the LEA, no ear advantage (NEA), and REA was measured for both tests in the NF condition. The prevalence of the REA shown by the black sections of the doughnuts in Figure 3A and B was greater when listeners were tested with the DNW test (78\%) than with the DWT (56\%). The prevalence of NEA (equal scores in the right and left ears) was much greater from the DWT at $23.2 \%$ than from the DNW test at $5.2 \%$.

Ear advantage scores calculated as the difference between the DOM and NDOM ears in the NF condition were again significantly different, $Z=-6.744, p<0.001$ between the DWT and the DNW test. As shown in Figure 2 , the value of ear advantage was significantly larger for the DNW test than for the DWT. The Friedman test was used to compare ear advantage scores calculated by the two methods (RE minus LE versus DOM minus NDOM) across the two different tests. Results confirmed that the ear advantage measures were significantly different, $\chi^{2}=$ $89.672(\mathrm{df}=3), p<0.001$. As shown in Figure 2, comparing black bars to gray bars for each test, the average value of ear advantage scores is always larger when calculated between DOM and NDOM ear because it removes the negative values of ear advantage from individuals who produce an LEA during the test. Also, ear advantage scores are significantly larger from DNW test results than from results obtained with the DWT.

\section{Comparisons between Test Versions}

The Kruskal-Wallis test was used to assess the differences in individual right and left ear results and ear advantage calculated between DOM and NDOM ears between the male and female versions of the DNW test. A total of 48 participants were tested with the female recording of the DNW test and 47 participants were tested with the male recording of the DNW test. Left ear results were not significantly different, but right ear scores were significantly higher for participants who listened to the female version of the test, $\chi^{2}=7.653(\mathrm{df}=1), p=0.006$. Average ear advantage scores were also larger for the participants who listened to the female version of the test, but the results narrowly missed achieving significance, $\chi^{2}=3.643(\mathrm{df}=3), p=0.056$.

\section{Confidence Intervals}

Bootstrapping methods were used to calculate the $95 \%$ confidence intervals for the DOM and NDOM ear scores and ear advantage scores from both tests. The low and high $95 \%$ criterion cutoff ranges for the DNW test were considerably wider than the $95 \%$ criterion cutoff ranges than the DWT. The low and high criterion cutoff ranges for the DOM and NDOM ears in the DNW test were $67.33-72.40$ and $48.25-54.32$, respectively. The low and high $95 \%$ criterion cutoff ranges for the DWT in the $\mathrm{NF}$ condition for the DOM and NDOM ears were 92.55-94.14 and 88.74-91.05, respectively. The presence

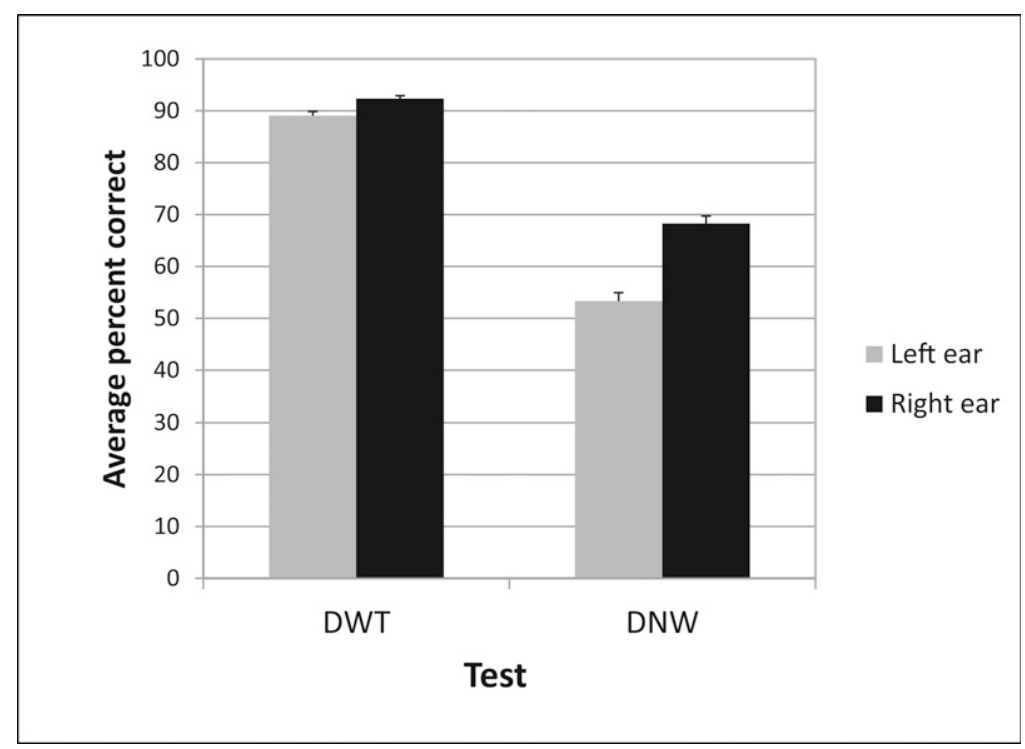

Figure 1. Scores within each test were higher for the right ear than for the left ear. Scores for both ears were significantly lower on the DNW test than with the DWT. 


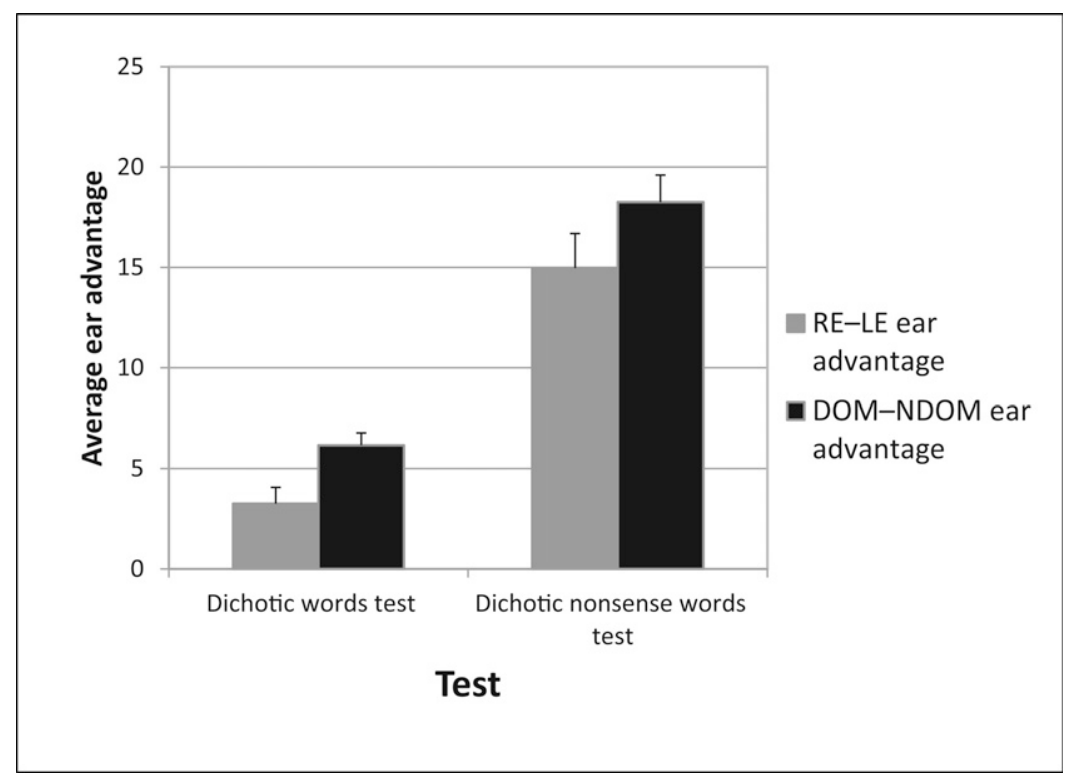

Figure 2. Ear-advantage scores from the DNW test were larger than those obtained from the DWT. This was true when measured by comparing right ear (RE) with left ear (LE) and when comparing DOM ear with NDOM ear.

of a wider and lower cutoff range may reduce the possibility of floor or ceiling effects when using the DNW test instead of the DWT.

Each participant's individual scores were compared with the low cutoff for the DOM and NDOM ear scores and to the high cutoff for ear advantage. When a score failed to reach a low criterion or exceeded a high criterion, it was marked. Marked scores from the two tests were compared within each individual participant and when the pattern of deficits matched according to a diagnostic rubric established for interpreting DL tests (Moncrieff et al, 2016), the individual's performance was interpreted to potentially represent the diagnostic categories of amblyaudia (AMB) and dichotic dysaudia (DD). AMB is defined as a large asymmetry between ears on dichotic tasks with either normal or below-normal performance in the DOM ear. DD was recently defined as bilateral weakness in dichotic-listening tasks with normal monaural performance in both ears (Moncrieff et al, 2016). Within the 95 participants, the scores from both tests of ten participants fit the pattern for the diagnosis of AMB. One participant's scores fit the pattern for AMB plus $\mathrm{DD}$, and one participant's scores fit the pattern for DD.

The high prevalence of abnormal performance distribution in this participant group was surprising. The high prevalence of scores outside the normal range may reflect a higher prevalence of weak binaural integration skills than expected among university students.

\section{Comparisons between NF and Directed Test Conditions}

As shown by the significantly poorer performance during the NF condition of the DNW test compared with the
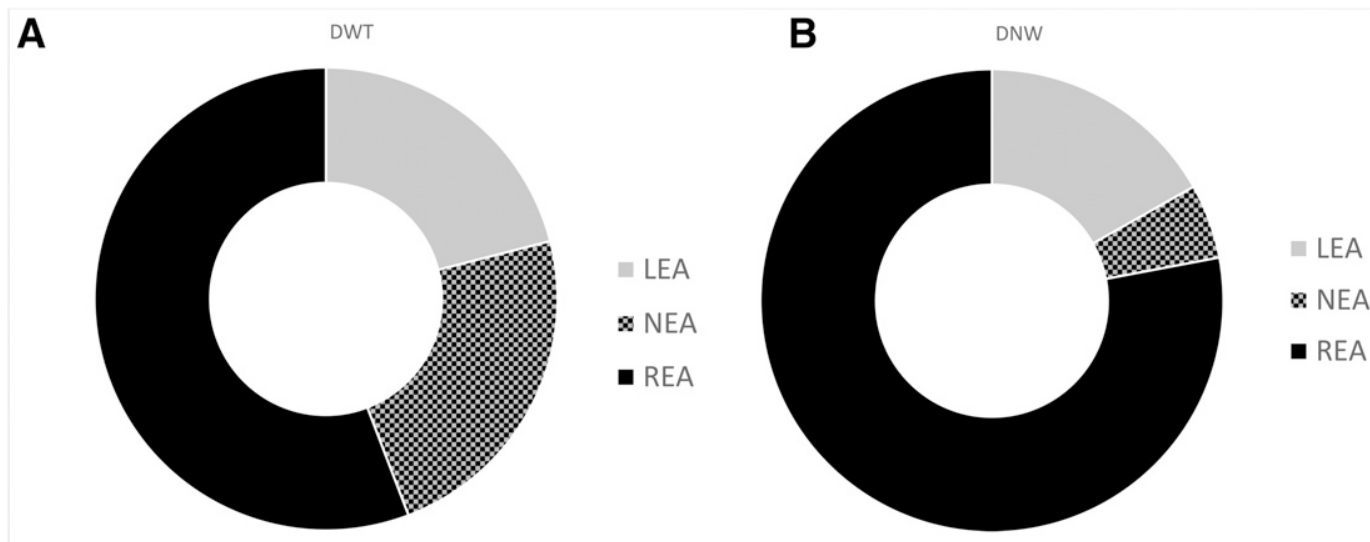

Figure 3. (A) In the DWT, the prevalence of LEA (20.8\%), REA (56\%), and NEA (23.2\%). (B) In the DNW test, the prevalence of LEA $(16.8 \%)$, REA $(78 \%)$, and NEA $(5.2 \%)$. 
DWT, nonsense words are generally more difficult to identify than are real words when presented in a binaural integration task. Both tests were also administered in selective attention formats during which it was hypothesized that their individual ear scores would increase. During two 25-word-pair lists from the DWT, participants were instructed to identify both words in a binaural integration task, but to identify the word heard in either the left or right ear first to selectively bias attention toward one ear at a time for half of the test. During two 25word-pair lists from the DNW test, participants were instructed to ignore one ear and to identify the nonsense word heard in the other ear in a binaural separation task. The Kruskal-Wallis test was used to compare right ear and left ear scores between the two listening conditions within each test. Neither the right nor left ear scores improved significantly in the directed response condition of the DWT, but during the selective attention format of the DNW test, scores were significantly different for both right ear scores, $\chi^{2}=73.825, p<0.001$, and left ear scores, $\chi^{2}=65.906, p<0.001$. As shown in Figure 4, participants performed at higher levels in both ears when they were allowed to direct their attention toward one ear and ignore the other ear. Despite the improvements in individual ear scores when participants could ignore one ear and focus attention on the other, there was no significant change in the values of their ear advantage scores whether the ear advantage was calculated in the traditional of RE minus LE manner, $\chi^{2}=0.450$, $p=0.502$ or in the alternative manner of DOM minus NDOM, $\chi^{2}=1.086, p=0.297$.

\section{DISCUSSION}

$\mathrm{T}$ he purpose of this study was to evaluate a newly created dichotic nonsense test without semanticity, and to compare scores achieved by young adults from the new test to performance on the DWT, a dichotic words test that contains both lexical and semantic

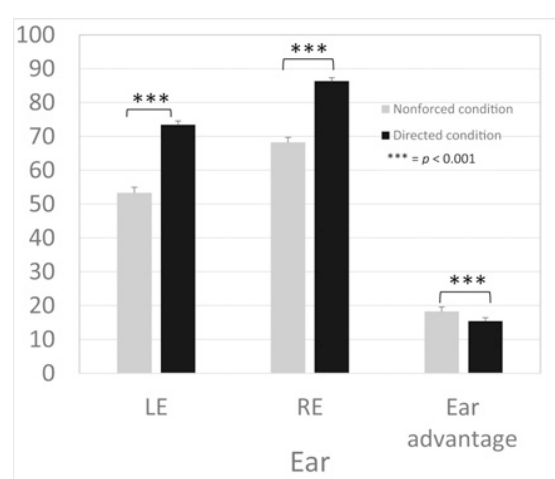

Figure 4. Participants performed at higher levels in both ears in the directed attention condition. There was no significant change in ear advantage values when calculated by RE minus LE manner, $\chi^{2}=$ $0.450, p=0.502$ or by DOM minus NDOM, $\chi^{2}=1.086, p=0.297$. content. Participants produced the same overall pattern of results when tested with the DNW test as when they were tested with the DWT, but scores were significantly lower for nonsense words from the DNW test in both ears. These results are similar to those shown by Findlen and Roup (2011) who also reported significantly poorer performance among young adults when they were tested with nonsense consonant-vowel-consonant syllables compared with their results with meaningful consonant-vowel-consonant words. They proposed that use of nonsense syllables during dichotic tests may provide means for assessing speech recognition without the interference of familiar vocabulary.

Results from both DNW test and DWT revealed higher average right-ear scores than left-ear scores so that a majority of listeners produced an REA. Although most participants produced an REA with both of these dichotic tests as expected, the prevalence of the REA was larger with the new DNW test than with the DWT. Significantly fewer participants produced either an LEA or NEA with this new test, suggesting that the absence of semanticity may increase the listeners' need to encode the phonological information from each target stimulus in a way that more effectively taps into language-dominant processes in the cortical left hemisphere. When presented with common single-syllable words that are part of a listener's vocabulary, listeners may use semantic familiarity to rapidly facilitate identification of words and reduce the demands required to process the incoming stimuli from either side, thereby improving performance for words presented to the NDOM left ear. This can be seen with the larger prevalence of LEA or NEA among typical listeners when they engage in standard DL tests (Hiscock et al, 1994). When the stimuli presented to both ears carry no semantic familiarity, the listener must engage auditory processes to encode the phonemic features of presentations to each side. In a typical left-hemisphere-dominant listener, it is presumed that phonological representations of these novel incoming stimuli are more actively processed on that side of the cortex, thereby giving preference to stimuli ascending from the contralateral right ear. This preference to stimuli arriving at the right ear could account for the higher prevalence of the REA when participants are presented with nonsense word stimuli.

Another explanation for a higher prevalence of the REA from DNW test results could be due to the effects of voiceonset time (VOT) for the stimulus pairs. It has been shown that when a word with a long VOT is presented to one ear at the same time that a word with a short VOT is presented to the other ear, the listener is much more likely to identify the word with the long VOT. This preference for the word with the long VOT can overcome the structural bias toward the ear that is contralateral to the listener's languagedominant hemisphere, thereby resulting in an LEA among listeners who generally produce an REA for dichotic stimuli (Rimol et al, 2006). The VOT of each nonsense word was 
not measured when creating the DNW test, but information about the relative VOT for each pair could help to explain the higher prevalence of the REA for this new test. The measurement and investigation of VOT through experimental design will be investigated thorough future study.

The magnitude of ear advantage scores was significantly larger on the DNW test, highlighting the increased difficulty of task. Whether the improved performance in the right ear during the DNW test was due to a more fully engaged left hemisphere to process unfamiliar verbal content or due to the effects of VOT between the two ears, it appears that the resulting magnitude of ear advantage reflects that the test taxes auditory processes and leads to a greater difference between the two ears. Because children learning in school are continually presented with new words that contain unfamiliar patterns of phonemes, the binaural integration processes engaged by the nonsense words in this DNW test may more accurately reflect the skills that are needed when children learn new words in school. As such, the test may prove to be a useful tool to assess children's ability to use binaural integration skills to decode new verbal material. The DNW test may be more sensitive to bottom-up sensory differences in processing capabilities between the two ears than a test containing greater semantic content that can be influenced by greater knowledge and cognitive control. In addition, the lack of semantic content in the DNW stimuli may present greater potential for use with nonnative English speakers, increasing its use as a test with "universal design."

It has been widely documented that individual right and left ear scores increase when listeners are instructed to selectively attend to stimuli presented toward one ear during dichotic tasks (Bryden et al, 1983; Obrzut et al, 1986; Hiscock et al, 1999). Researchers investigating the effects of attentional bias noted that the degree to which scores in each ear are enhanced depends on an interaction between attention and stimulus characteristics (Hiscock and Kinsbourne, 2011). Results from this study were consistent with evidence that directed attention during a task with highly familiar vocabulary words did not alter performance in a significant manner but that selective attention toward novel nonsense words did significantly improve performance in each ear. In both the cases, the magnitude and direction of ear advantage scores were unchanged with the addition of attentional controls. Had attention influenced these results, a change in the magnitude of the ear advantage would have been evident in ear advantage calculated between scores in the DOM and NDOM ears and changes in the direction of the ear advantage would have been evident in the value of ear advantage calculated between scores in the right and left ears. Both of those values were not significantly different across the two versions of the test administration for either test. It should be noted that the DWT was always completed before the DNW test, with the purpose of familiarizing to the task. There is a chance that this familiarity influenced the results, so repetition of this study with the DNW test completed first would help to confirm results.

When individual participant scores were compared within each test with the 95th percentile cutoff score, results were remarkably similar within each test. The same number of individuals (33) produced weakness in the NDOM ear from one test that might suggest AMB, but an individual must produce the same pattern of weakness across two DL tests before the suggestion of AMB can be made. Only 11 individuals showed a consistent pattern with weakness in the NDOM ear during both tests that might suggest AMB. Twice as many individuals demonstrated weakness in the DOM ear during the DNW test (34) than during the DWT (17), but only two showed that pattern across both tests that could suggest DD. It should also be noted that more than twice as many participants' scores fell below the cutoff criterion for the DNW test than for the DWT. These results demonstrate the critical need to base referrals and diagnoses on no fewer than two different tests of DL. Here, the DNW test has the potential to supplement diagnostic information and aid in differential diagnosis, but the test should always be used as part of a test battery. Because twice as many participants' scores fell below the cutoff criterion for the DNW test than the DWT, the cutoffs measured here are not recommended as normative data. The data are observational and may reflect the limited pool of graduate university students. Future study should include a wider variety of participants.

Tests differ in lexical content, duration, and influence of attention, all of which can have an impact on binaural integration skills. The diagnosis of AMB or DD should never be made on the basis of only one test, but should be suggested when a listener produces the same pattern of results across a minimum of two DL tests. A dichotic test creates an unfamiliar and challenging listening task for most listeners whose performance can improve with practice and shift from falling below a critical cutoff value to being within the limit of normal. Therefore, test order may have played a part in individual test performance whereby participants did more poorly on the first test they were given and then performed at a normal level on the second test after having some practice with DL. Furthermore, individual listeners show some variability across tasks with different stimulus types when tested with syllables, single-syllable vocabulary words, and the words that represent digits that could lead to lower than normal performance on one test.

Another factor that could have resulted in lower performance on one test than on the other is the gender of the voice presenting the target stimuli. Certain factors can be associated with the ability to perceive a voiced stimulus, one of these being fundamental frequency. The use of a single talker as compared to more than one talker over several conditions can lead to 
decreased word-recognition performance (Mullennix et al, 1989). Therefore, presenting stimuli with a single talker throughout all test conditions or using a talker of the same gender may lead to better perception. In the present study, half of the listeners heard two different male voices between tests, and the other half of participants heard a male voice in the DWT and a female voice in the DNW test. Across all participants, those who heard the female version of the DNW test performed at a higher level in their DOM ears, so it is possible that those whose DOM ear score fell below the normal cutoff value may have been listening to the male version of the DNW test. This was not the case, however. The same number of participants produced lower than normal performance in their DOM ears when listening to the female version of the test as when listening to the male version of the test, so it is not likely that gender of the voice had an impact on performance values.

Another advantage to the DNW test is that average performance was significantly lower and more variable than with the DWT. This suggests that the DNW test as developed for this study may be useful as a complement to tests with low semantic content, such as tests with digits, that produce "ceiling effects" in adult and older child participants (Moncrieff and Musiek, 2002; Neijenhuis et al, 2002). Audiologists report that the DL test is the most widely used assessment tool in their auditory processing battery (Emanuel et al, 2011). The DNW test may be considered as a useful part of that battery for several reasons. Because it mimics the listening situations and processing strategies faced by children during routine learning experiences in school as they are acquiring vocabulary, it may provide important information about binaural integration skills separate from a listener's established lexicon. The presence of multiple lists provides the clinician with a means to assess binaural integration and binaural separation abilities, thereby enabling a measure of a listener's ability to follow directions and engage in selective attention. Confidence intervals around the 95th percentile are useful in establishing guidelines for interpretation of test results. Information from this study is useful for evaluating young-adult females, but a follow-up study of male and female children between the ages of 6 and 18 is needed to calculate confidence intervals for children and to investigate the appropriateness for the test in this population.

\section{REFERENCES}

Bellis TJ. (2002) Developing deficit-specific intervention plans for individuals with auditory processing disorders. Semin Hear 23: 287-295.

Boothroyd A, Nittrouer S. (1988) Mathematical treatment of context effects in phoneme and word recognition. J Acoust Soc Am 84(1):101-114.
Bryden MP, Munhall K, Allard F. (1983) Attentional biases and the right-ear effect in dichotic listening. Brain Lang 18(2):236-248.

Cherry EC. (1953) Some experiments on the recognition of speech, with one and two ears. J Acoust Soc Am 25:975-979.

DeBonis DA, Moncrieff D. (2008) Auditory processing disorders: an update for speech-language pathologists. Am J Speech Lang Pathol 17(1):4-18.

Emanuel DC, Ficca KN, Korczak P. (2011) Survey of the diagnosis and management of auditory processing disorder. Am J Audiol 20(1):48-60.

Findlen UM, Roup CM. (2011) Dichotic speech recognition using CVC word and nonsense CVC syllable stimuli. J Am Acad Audiol 22(1):13-22.

Haukoos JS, Lewis RJ. (2005) Advanced statistics: bootstrapping confidence intervals for statistics with "difficult" distributions. Acad Emerg Med 12(4):360-365.

Henderson AR. (2005) The bootstrap: a technique for data-driven statistics. Using computer-intensive analyses to explore experimental data. Clin Chim Acta 359(1-2):1-26.

Hiscock M, Cole LC, Benthall JG, Carlson VL, Ricketts JM. (2000) Toward solving the inferential problem in laterality research: effects of increased reliability on the validity of the dichotic listening right-ear advantage. J Int Neuropsychol Soc 6(5):539-547.

Hiscock M, Inch R, Jacek C, Hiscock-Kalil C, Kalil KM. (1994) Is there a sex difference in human laterality? I. An exhaustive survey of auditory laterality studies from six neuropsychology journals. $J$ Clin Exp Neuropsychol 16(3):423-435.

Hiscock M, Inch R, Kinsbourne M. (1999) Allocation of attention in dichotic listening: differential effects on the detection and localization of signals. Neuropsychology 13(3):404-414.

Hiscock M, Kinsbourne M. (2011) Attention and the right-ear advantage: what is the connection? Brain Cogn 76(2):263-275.

Hugdahl K, Andersson L. (1986) The "forced-attention paradigm" in dichotic listening to $\mathrm{CV}$-syllables: a comparison between adults and children. Cortex 22(3):417-432.

Hugdahl K, Carlsson G, Eichele T. (2001) Age effects in dichotic listening to consonant-vowel syllables: interactions with attention. Dev Neuropsychol 20(1):445-457.

Keith RW, Katbamna B, Tawfik S, Smolak LH. (1987) The effect of linguistic background on staggered spondaic word and dichotic consonant vowel scores. $\mathrm{Br}$ J Audiol 21(1):21-26.

Kimura D. (1961) Cerebral dominance and the perception of verbal stimuli. Can J Psychol 15:166-171.

Kimura D. (1967) Functional asymmetry of the brain in dichotic listening. Cortex 3:163-178.

Moncrieff D. (2015) Age- and gender-specific normative information from children assessed with a dichotic words test. J Am Acad Audiol 26(7):632-644.

Moncrieff D, Keith W, Abramson M, Swann A. (2016) Diagnosis of amblyaudia in children referred for auditory processing assessment. Int $J$ Audiol 55(6):333-345.

Moncrieff DW. (2006) Identification of binaural integration deficits in children with the competing words subtest: standard score versus interaural asymmetry. Int $J$ Audiol 45(4): 200-207, discussion 207-210. 
Moncrieff DW. (2011) Dichotic listening in children: age-related changes in direction and magnitude of ear advantage. Brain Cogn 76(2):316-322.

Moncrieff DW, Musiek FE. (2002) Interaural asymmetries revealed by dichotic listening tests in normal and dyslexic children. J Am Acad Audiol 13(8):428-437.

Moncrieff DW, Wilson RH. (2009) Recognition of randomly presented one-, two-, and three-pair dichotic digits by children and young adults. $J$ Am Acad Audiol 20(1):58-70.

Mullennix JW, Pisoni DB, Martin CS. (1989) Some effects of talker variability on spoken word recognition. J Acoust Soc Am 85(1): 365-378.

Musiek FE, Shinn J, Hare C. (2002) Plasticity, auditory training, and auditory processing disorders. Semin Hear 23:263-275.

Neijenhuis K, Snik A, Priester G, van Kordenoordt S, van den Broek P. (2002) Age effects and normative data on a Dutch test battery for auditory processing disorders. Int J Audiol 41(6):334-346.

Nittrouer S, Boothroyd A. (1990) Context effects in phoneme and word recognition by young children and older adults. J Acoust Soc Am 87(6):2705-2715.
Noffsinger D, Martinez CD, Andrews M. (1996) Dichotic listening to speech: VA-CD data from elderly subjects. J Am Acad Audiol $7(1): 49-56$.

Noffsinger D, Martinez CD, Wilson RH. (1994) Dichotic listening to speech: background and preliminary data for digits, sentences, and nonsense syllables. J Am Acad Audiol 5(4):248-254.

Obrzut JE, Boliek CA, Obrzut A. (1986) The effect of stimulus type and directed attention on dichotic listening with children. $J$ Exp Child Psychol 41(1):198-209.

Rimol LM, Eichele T, Hugdahl K. (2006) The effect of voice-onsettime on dichotic listening with consonant-vowel syllables. Neuropsychologia 44(2):191-196.

Roup CM, Wiley TL, Wilson RH. (2006) Dichotic word recognition in young and older adults. J Am Acad Audiol 17(4):230-240, quiz $297-298$.

Techentin C, Voyer D. (2011) Word frequency, familiarity, and laterality effects in a dichotic listening task. Laterality: asymmetries of body. Brain $\operatorname{Cogn}$ 16:313-332.

Yost WA. (2013) Fundamentals of Hearing: An Introduction. Boston, MA: Brill. 